\title{
Safety profile of dextran-spermine gene delivery vector in mouse lungs
}

\begin{abstract}
A nano-sized polymer, dextran-spermine (D-SPM), was shown to have the capacity to deliver gene to the lung of mouse via intranasal route. In this study, assessments on the safety profile of D-SPM were performed to complement the gene expression results. African green monkey kidney fibroblast (COS-7) and human adenocarcinoma breast (MCF-7) cells transfected with D-SPM/pDNA showed massive reduction in the number of viable cells. As for in vivo study, elevated level of neutrophils was observed, despite the minimal level of pro-inflammatory cytokines (TNF- $\alpha$, IL-12, IFN- $\gamma$ detected in the bronchoalveolar lavage fluid (BALF) of mice treated with the D-SPM/pDNA complexes. Histology profile examinations of the lungs showed mild inflammatory responses, with inflamed areas overlap with healthy areas. Although reduction of mice weight was seen at day 1 post administration, the mice did not show any sign of abnormal behavior or physical appearance. Biodistribution study was performed to determine the ability of the D-SPM/pDNA complexes to infiltrate to other nonintended organs. The result showed that the D-SPM/pDNA complexes were only localized at the lung and no gene expression was detected in other organs or blood. In short, these results indicate that the D-SPM/pDNA exhibited mild toxicity in the mouse lungs.
\end{abstract}

Keyword: Dextran-spermine; Mouse lung; Non-viral delivery; Polymer; Safety 